\title{
A New Approach: Patient Portals for Primary Intervention
}

\author{
Cody Jackson, $\mathrm{BSc}^{1}$, Renée Bradley ${ }^{1}$
}

${ }^{1}$ Faculty of Medicine, University of Ottawa

\section{A B STRACT}

Patient portal use in Canada is still quite new, and there has been little research conducted on its effectiveness within the Canadian healthcare system. However, by observing current portals in use internationally, as well as analyzing pilot studies, we can anticipate many benefits to patient health. Increasing evidence shows that portals have the potential to make a significant impact on patient health. Current portal functions, as well as functions yet to be implemented, have the capacity to increase the overall efficiency of the healthcare system.

\section{RÉSUMÉ}

L'utilisation de portails est encore assez récente chez les patients au Canada et peu de recherche n'a été effectuée sur leur efficacité dans le système de santé canadien. Or, l'observation des portails actuellement utilisés sur le plan international et l'analyse d'études pilotes nous permettent d'anticiper de nombreux avantages pour la santé des patients. II apparaît de plus en plus clairement que les portails pourraient avoir des répercussions importantes sur la santé des patients. Les fonctions que l'on retrouve actuellement sur les portails, de même que celles à venir, ont la capacité d'accroître l'efficacité globale du système de santé.

Many healthcare professionals believe that for the Canadian healthcare system to remain fiscally viable, there needs to be a greater focus on both intervention before disease-onset, and on keeping patients from unnecessarily using expensive healthcare resources [1]. In terms of cost-effectiveness and maintenance of patient quality of life, the significance of primary intervention cannot be overstated.

There are several approaches that help family physicians prevent exacerbations and disease progression. One such method that intervenes early on, but is not currently well-utilized, is online patient portals. A patient portal is an electronic medium accessible via the Internet, similar to a website, which is connected to family physician electronic medical records (EMRs). There are defining traits that distinguish patient portals from EMRs. EMRs simply allow a patient's personal medical record and the entirety of their medical history to be recorded digitally. These records may remain solely within medical centres or institutions, but also have the capacity to be shared externally. The records are not usually readily accessible to patients. Conversely, portals allow patients to track their health, connect to physicianrecommended resources, access physician instructions, see their list of prescriptions and advice, book appointments and take advantage of countless other functionalities [2]. For physicians, portals have the ability to improve the management of medical offices by increasing patient contact and easing workflow.

There has been some recent advancement in the field of patient portals, particularly in the United States. Some patients

Keywords: patient portals, EMR, primary intervention in the United States have access to versions of patient portals, mainly through their health insurance providers, wherein they can log certain health information, book appointments and perform some other services online [3]. Within Canada, there are limited examples of fully developed portals in widespread use, while worldwide there has been relatively little study of them $[4,5]$. Due to the lack of research on patient portals, discussion on this topic is largely speculative. Systems such as McMaster's MyOSCAR and Sunnybrook Hospital's MyChart have taken the first steps towards making patient portals and personal health records a standard for the Canadian healthcare system, but there are still many functions with great potential that are currently not in use $[6,7]$. This paper will provide a general overview of the purpose of patient portals, and provide an editorial on some functions that are already in use by the portals developed today and other functions that are yet to be implemented. However, many of the specific technical details as well as how these portals will eventually be implemented will not be addressed.

Patient portals do not provide new information, nor do they change treatments or guidelines for patients; they are simply a new mechanism of communication and education. While only $11 \%$ of patients report that their first line of inquiry is their primary care physician, a noteworthy $49 \%$ state that the Internet is their first resource [8]. Portals have shown the capacity to reduce adverse events in patients who require chronic care. For example, a study conducted at the University of Washington General Internal Medicine Clinic found that type II diabetes patients receiving portal benefits such as web-based care management and a shared EMR had overall better glycemic control [9].

Portals also enable physicians to become better health 
advocates by tapping into a medium that most patients are already using, and by encouraging patients to get involved in maintaining their own health. According to a recent health informatics study in the U.S., health information is sought online by $81 \%$ of Internet users and $66 \%$ of all adults. Even more significant is that among these users, $78.5 \%$ indicate that the health information they find online has changed the way they think about health [10].

Web-based portals could be immensely helpful to patients with chronic disease and those with complicated medical histories $[9,11]$. Portals would enable patients to monitor their blood pressure, caloric intake, weight, blood glucose, symptom frequency, lipid levels, and more, showing health trends and potentially providing better motivation to manage needs [12]. Portals have the potential to be used by doctors to recommend specific dietary and exercise regimes and to link patients to approved health resources and frequently asked questions. Some current systems allow patients to contact nurses and physicians via portal mail for quick questions not requiring an office visit and to book appointments online, easing their frustration at trying to reach someone during set hours. This new tool also allows patients to input notes before their appointment, which would then be available in office, and to have easy access to a list of their medication names, symptoms and questions $[6,7]$.

It would be interesting to further study portals and their effect on patient-doctor communication with sensitive information. Portals may prove to be a more comfortable means of communication for patients who may be reluctant to bring up sensitive information, such as weight, abuse or sexual issues, in person, by allowing patients to electronically send concerns to their physician. These functions may allow patients to better address their medical problems autonomously and permit healthy patients to observe negative trends in their health so they are able to intervene before disease onset [13]. Portals could also help patients recognize the signs of drug misuse and poor chronic disease control by providing a resource that outlines possible warning signs.

One of the most significant possible benefits of patient portals could be the ability to help patients distinguish between symptoms needing medical attention and those simply needing conservative treatment at home. Often those with chronic disease are unsure of "red flag" symptoms, which can even be painless in the case of lower limb ulcer infections in diabetics. This information dissemination could be accomplished by having a section, divided by disease/system, showing symptoms that should elicit a physician visit versus those that can be treated symptomatically. This would not only spare the healthcare system from unnecessary visits, but promote early intervention to prevent disease progression. It also has the potential to reduce the frequency of disease exacerbations by ensuring that patients are knowledgeable about potential preventative measures and are alert to warning signs. According to a study conducted in Virginia family practices, the increase in the percentage of patients who were up-to-date on preventative services was double among those with portal access compared to a control group without access [14]. Not only do portals have the potential to help those with established disease, but they can help healthy patients monitor their health and prevent the onset of disease by providing them with knowledge of preventative services and resources, such as health guidelines and recommended lifestyle goals.

Patient portals allow all of this information to be in one easily found and frequently visited location. Another immensely useful function is the ability for other family physicians or specialists to access patient health information with the permission of the patient. Currently the transfer of information from hospital to family clinic and specialist to general practitioner is normally limited to a single letter or a scanned handwritten hospital note that is often illegible [15]. Current medications, past procedures, clinical findings, notes, lab reports, differentials and other medical information, all have the potential to be shared by simply accessing a website (portal), with patient consent. Better information sharing can create a more cohesive interdisciplinary health team where each physician, healthcare worker and patient is better informed of the relevant health information and where communication between staff is facilitated [16].

One of the major challenges of implementing change to any healthcare system is that one must overcome skepticisms and concerns. The introduction of patient portals is no exception to this. As one would expect, younger patients seem to be more comfortable with patient portals, which may be attributed to better computer literacy and familiarity with technology [17]. Fortunately, portals have the potential to include functions such as text-to-read and font size modification which may increase the ease of use for older users and those with hearing or vision impairments. Another potential problem with patient portals is that they will allow patients to receive much more information than they are accustomed to, via the portal, which may create unnecessary anxiety and worry among patients. What must be stressed, however, is that the portal is not necessarily a continuation of the physician's EMR, but a derivative of it; only information that has been approved by the physician will be available on the portal. Similarly, specific notes and lab tests could be set up to only be accessible to certain accounts such as that of the primary care physician or another healthcare worker. This would prevent certain information from being read by patients before their physician has had time to discuss it with them. This would also allow the doctor to control which notes are seen by the patient, in cases where the physician believes his personal notes may cause harm, such as with mental health patients [18]. Along with these concerns, issues of confidentiality must always be addressed when personal information is stored online. Surprisingly, this has shown to be a minor concern amongst patients using portals. During one study, a group of outpatients were given online access to their medical records. After surveying the patients, it was shown that only $3 \%$ were concerned about patient confidentiality [19]. Most portals currently in use have a username and password protection system that limits user access 


\section{Commentary}

to confidential patient information to the patient, as well as to others the patients chooses to share their information with, including family members and other doctors $[6,7]$. With regards to use by other physicians, one feasible system not currently in use to increase security is one in which all physicians have access to a central patient portal system but cannot access specific patient files unless given a patient-controlled personal access code.

Ultimately, portal use could have diverse and wideranging effects, with the goal of improving patient health literacy and communication. Portals also work to improve cooperation between physicians, and between physicians and their patients, as well as reducing patient over-reliance on costly healthcare resources. The Canadian healthcare system needs to focus on primary intervention to reduce burden on the system, and this is where patient portals are most effective. Patient portals have the potential to both advance patients' understanding of their own health needs, and improve their therapeutic relationship with their physician. The result of incorporation of portals will be a more efficient and cost-effective healthcare system.

\section{REFERENCES}

1. Kitts, J. More Cost-Effective Hospitals [Internet]. Ottawa, ON: Ottawa Citizen; 2013 Dec 16 [cited 2014 Aug 16]. Available from http://www2.canada. com/ottawacitizen/news/archives/ story.html?id=e678a959-7f0a-4426852b-195cb0103974\&p=1.

2. What is a patient portal? [Internet]. Washington, D.C., U.S. Department of Health and Human Services; [cited 2014 May 08]. Available from: http:// www.healthit.gov/providers-professionals/faqs/what-patient-portal.

3. My Health Manager [Internet]. [CA]: Kaiser Permante; c2013 [cited 2014 July 13]. Available from: https://healthy.kaiserpermanente.org/health/ care/consumer/my-health-manager.

4. Ross SE, Lin CT. The effects of promoting patient access to medical records: a review. J Am Med Inform Assoc. 2003; Apr;10(2):129-138.

5. Goldzweig CL, Orshansky G, Paige NM, et al.: Electronic patient portals: evidence on health outcomes, satisfaction, efficiency, and attitudes: a systematic review. Ann Intern Med 2013;159:677-687.

6. MyChart [Internet]. Toronto (ON): Sunnybrook Health Sciences Centre; c2014 [cited 2014 July 14]. Available from: http://sunnybrook.ca/ content/?page=mychartlogin-learnmore.

7. What is MyOSCAR [Internet]. Hamilton (ON): Mcmaster University; c2011 [cited 2014 July 10]. Available from: http://myoscar.org/about-myoscar.

8. Hesse BW, Nelson DE, Kreps GL et al. Trust and sources of health information: the impact of the Internet and its implications for healthcare providers: findings from the first Health Information National Trends Survey. Arch Intern Med 2005; 165: 2618-2624.

9. Ralston JD, Hirsch IB, Hoath J, Mullen M, Cheadle A, Goldberg HI. Webbased collaborative care for type 2 diabetes: a pilot randomized trial. Diabetes Care. 2009;32:234-9.

10. Shaw R and Johnson CM . Health Information Seeking and Social Media Use on the Internet among People with Diabetes. Online J Public Health Inform 2011; Vol.3: No. 1.

11. Osborn CY, Mayberry LS, Mulvaney SA, et al.: Patient web portals to improve diabetes outcomes: a systematic review. Curr Diabetes Rep; 2010;10:422435.

12. Tang P and Newcomb C. A Guide for Providing Patient Health Information. J Am Med Inform Assoc. 1998;5:563-570.

13. Bronson DL, Rubin AS, Tufo HM. Patient education through record sharing. Qual Rev Bull 1978;4:2-4.

14. Krist $\mathrm{AH}$, Woolf $\mathrm{SH}$, Rothemich SF et al. Interactive preventive health record to enhance delivery of recommended care: a randomized trial. Ann Fam Med. 2012;10:312-319.

15. Rodriguez-Vera F, Marin Y, Sanchez A et al. Illegible handwriting in medical records. J R Soc Med. 2002;95:545-546.

16. Essex B, Doig R, Renshaw J. Pilot study of records of shared care for people with mental illnesses. Br Med J 1990;300:1442-1446.

17. Cho AH, Arar NH, Edelman DE, Hartwell PH, Oddone EZ, Yancy WS Jr. Do diabetic veterans use the Internet? Self-reported usage, skills, and interest in using My HealtheVet Web portal. Telemed J E Health. 2010;16:595-602.

18. Roth LH, Wolford J, Meisel A. Patient access to records: tonic or toxin? Am J Psychiatry1980;137:592-596.

19. Jones RB, McGhee SM, McGhee D. Patient on-line access to medical records in general practice. Health Bull (Edinb). 1992;50:143-150. 